\title{
THE DESIGN OF SHIP AUTOPILOT BY APPLYING OBSERVER - BASED FEEDBACK LINEARIZATION
}

\author{
Zenon Zwierzewicz, Prof. \\ Maritime University of Szczecin, Poland
}

\begin{abstract}
The paper considers the problem of ship autopilot design based on Bech's model of the vessel. Since the model is highly nonlinear and some of the state vector coordinates are unavailable, the control system synthesis is performed by means of an output feedback linearization method combined with a nonlinear observer. The asymptotic stability of the overall system has been proven, including the asymptotic stability of the system internal dynamics. The performed simulations of the ship course-changing process have confirmed a high performance of the proposed controller. It has been emphasized that for its practical usability the system robustification is necessary.
\end{abstract}

Keywords: ship autopilot design, feedback linearization, nonlinear observer

\section{INTRODUCTION}

The course-keeping and course-changing problems are still vital issues during the ship handling process. In real circumstances, we have to cope with the presence of different kinds of uncertainty, such as: inaccuracies in the system model, the presence of random processes' statistics, such as winds, waves, currents, and other exogenous effects, the different sailing conditions such as speed, loading conditions, trim etc, as well as varied sailing routes - in open sea (deep water) or coastal (shallow waters) with a possible change in the under keel clearance.

This necessitates, when designing a vessel control system, employment of the techniques that take into account the process nonlinear effects as well as the consideration of the ship model parametrical uncertainty.

The most common methods for nonlinear systems control, intensively developed during last two decades, are feedback linearization and back-stepping. Each of them has its advantages and drawbacks.

The back-stepping procedure requires, for instance, the systems with special triangular structure (pure feedback form) and suffers from inherent 'explosion of terms' issue [7].

The feedback (or exact) linearization, in turn, has to satisfy the so-called matching condition [6], which implies that the uncertainty terms appear in the same equations as the control inputs $\mathrm{u}$, and as a result they can be handled by the controller.

Further issues that concern the above mentioned nonlinear control techniques relate to the model parametric uncertainty problem, as well as to the question of accessibility of the system state, which leads to the subsequent task of observer design.

Applying these techniques to the systems with uncertain parameters leads to the inexact compensation of the model non-linearities, which requires employing an adaptive or robust control methods during the controller design.

The main objective of the paper is to propose a ship coursekeeping controller design based on highly nonlinear Bech's ship model[4]. Because of the general model structure that excludes the use of the back-stepping method, we apply the feedback linearization combined with a nonlinear observer. It has been proven that the overall linearized system as well as its internal (zero) dynamics $[6,13]$ are asymptotically stable.

The herein proposed design assumes the full knowledge about the model parameters. However, knowing that for its practical usability the system parametrical uncertainties have to be considered, the paper is the first part of a larger project. The adaptive version of this proposal will be presented in its second part.

The paper is divided into five sections and ends with conclusions. The second section presents the models of the ship and steering gear, including model parameters. In the third section the controller design by using output feedback linearization is described along with an analysis of system internal dynamics. In the fourth section the reduced - order nonlinear observer is derived and the stability of the overall system is proven. The last, fifth section includes a short description of simulation tests and their results.

\section{MODELS OF THE SHIP AND ITS STEERING GEAR}

At first we introduce the following Bech's ship dynamic model $[4,1,15]$

$$
\dddot{\psi}+\left(\frac{1}{T_{1}}+\frac{1}{T_{2}}\right) \ddot{\psi}+\frac{K}{T_{1} T_{2}} H_{B}(\dot{\psi})=\frac{K}{T_{1} T_{2}}\left(\delta+T_{3} \dot{\delta}\right)
$$


where

$\psi(\mathrm{t})$ - ship heading - the controlled variable

$\dot{\psi}(\mathrm{t})=\mathrm{r}$ - angular velocity (rate of turn)

$\delta(t)$ - rudder deflection - a control variable

Function $H_{B}(\dot{\psi})$ describes a nonlinear ship maneuvering characteristic. In the steady state when $\ddot{\psi}(\mathrm{t})=\ddot{\psi}(\mathrm{t})=\dot{\delta}(\mathrm{t})=0$, it follows that $\delta=\mathrm{H}_{\mathrm{B}}(\dot{\psi})$, which is the formula describing Bech's reversed spiral characteristic. A good approximation for the non-linear function $\mathrm{H}_{B}(\dot{\psi})$ has appeared to be :

$$
H_{B}(\dot{\psi})=b_{3} \dot{\psi}^{3}+b_{2} \dot{\psi}^{2}+b_{1} \dot{\psi}+b_{0}
$$

A single screw propeller or asymmetry in the hull will cause a non-zero value of $b_{0}$. Similarly, symmetry in the hull implies that $b_{2}=0$. Course instability results in a negative value of $b_{1}$. Since a constant rudder angle is required to compensate for constant steady-state wind and current disturbances, the bias term $b_{0}$ is frequently taken as null, being conveniently treated as an additional rudder offset.

As the ship model nominal parameters, the dynamic maneuvering parameters of marine class vessel [3] are adopted: $\mathrm{K}=11,11 / \mathrm{min}, \mathrm{T}_{1}=1,967 \mathrm{~min}, \mathrm{~T}_{2}=0,13 \mathrm{~min}, \mathrm{~T}_{3}=0,308 \mathrm{~min}$, $\mathrm{b}_{3}=0,4 \mathrm{~min} 3 / \mathrm{rad} 2, \mathrm{~b}_{2}=0 \min 2 / \mathrm{rad}, \mathrm{b}_{1}=2 \mathrm{~min}, \mathrm{~b}_{0}=0 \mathrm{rad}$.

The ship has the following characteristics: displacement: $18541 \mathrm{~m} 3$, draft: $8.23 \mathrm{~m}$, length overall: $171,8 \mathrm{~m}$, length between perpendiculars: $160,93 \mathrm{~m}$, maximum beam: $23,17 \mathrm{~m}$, one propeller, and maximum speed: 15 knots. The maximum rudder angle and maximum rate of turn are: $35 \mathrm{deg}=0,61 \mathrm{rad}$ and $1 \mathrm{deg} / \mathrm{s}=1,047 \mathrm{rad} / \mathrm{min}$, respectively.

In the control synthesis process a steering gear model should also be taken into account (Fig. 1).

Ignoring the action of the rudder limiter, the steering gear dynamics is given by the simple formula

$$
\dot{\delta}=-\delta+\delta_{z}
$$

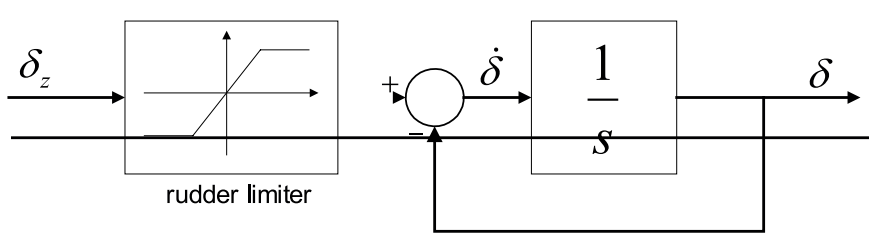

Fig. 1. Block diagram of the steering gear

When the models (1) and (3) are combined and written in the state-space form, the overall ship model is as follows:

$$
\begin{aligned}
& \left\{\begin{array}{l}
\dot{x}_{1}=x_{2} \\
\dot{x}_{2}=x_{3}+c x_{4} \\
\dot{x}_{3}=-a x_{3}-b H\left(x_{2}\right)+e x_{4} \\
\dot{x}_{4}=-x_{4}+u
\end{array}\right. \\
& y=x_{1}
\end{aligned}
$$

where :

$\mathrm{x}_{1}=\psi ; \mathrm{x}_{2}=\dot{\mathrm{x}}_{1}=\dot{\psi} ; \mathrm{x}_{3}=\ddot{\psi}-\mathrm{c} \delta ; \mathrm{x}_{4}=\delta ; \mathrm{u}=\delta_{\mathrm{z}}$

and

$a=\frac{T_{1}+T_{2}}{T_{1} T_{2}}, b=\frac{K}{T_{1} T_{2}}, c=b T_{3}=\frac{K T_{3}}{T_{1} T_{2}}, e=b-a c$ (6)

\section{CONTROLLER DESIGN BY APPLYING OUTPUT FEEDBACK LINEARIZATION}

The problem of course-keeping is a task of designing an automatic control aid (autopilot) which is able, by using appropriate rudder actions, to control the ship motion as to maintain a pre-assigned constant heading. This problem is often considered as consisting of two sub-problems. The first one concerns the ship control along a straight line with small course deviations. The second problem concerns the proper ship control during large manoeuvres, i.e. the problem of the quality of transition process as a response to a course step-change.

\section{SYSTEM TRANSFORMATION}

To design our controller we apply the input-output feedback linearization method $[5,6,10]$. Avoiding the complicated formalism of Lie derivatives, we simply repeatedly differentiate the output $\mathrm{y}$ in respect to time until the control $\mathrm{u}$ appears in the subsequent equation:

$$
\begin{aligned}
& \dot{y}_{1}=y_{2} \\
& \dot{y}_{2}=y_{3} \\
& \dot{y}_{3}=\underbrace{-a x_{3}-b H\left(x_{2}\right)+(e-c) x_{4}}_{f(\boldsymbol{x})}+\underbrace{c}_{g(\boldsymbol{x})} u
\end{aligned}
$$

where the new coordinates are:

$$
\mathrm{y}_{1}=\mathrm{y}=\mathrm{x}_{1} ; \mathrm{y}_{2}=\dot{\mathrm{y}}=\mathrm{x}_{2} ; \mathrm{y}_{3}=\ddot{\mathrm{y}}=\mathrm{x}_{3}+\mathrm{cx}_{4}
$$

Now, we can get the desired controller by applying the prefeedback (9) that leads to the cancellation of nonlinear terms of the model (7) [14]:

$$
u=\frac{-f(\boldsymbol{x})+v}{g(\boldsymbol{x})}
$$

where:

$v=k_{1}\left(y-y_{d}\right)+k_{2} \dot{y}+k_{3} \ddot{y}=k_{1}\left(x_{1}-y_{d}\right)+k_{2} x_{2}+k_{3}\left(x_{3}+c x_{4}\right)$

Inserting the controller (10),(9) to the system (7), we get the linear system

$$
\dddot{y}-k_{3} \ddot{y}-k_{2} \dot{y}-k_{1}\left(y-y_{d}\right)=0
$$

or

$$
\dddot{\tilde{y}}-k_{3} \ddot{\tilde{y}}-k_{2} \dot{\tilde{y}}-k_{1} \tilde{y}=0
$$


where:

$$
\tilde{y}=y-y_{d}
$$

This system is stable by virtue of the proper choice of coefficients, which can be done by, e.g., the pole placement technique.

\section{INTERNAL DYNAMICS ANALYSIS}

By means of input-output linearization, the dynamics of nonlinear system is decomposed into an external (inputoutput) dynamics part and an internal ('unobservable') part.

After the partial coordinate transformation (8), we have got a new system (7) of third order which represents the external dynamics. Because the original system (4) is of fourth degree here, there must be an additional dynamics [6] (internal or zero dynamics) described by a subsequent equation.

It is very important, during the controller design, to find out whether the zero dynamics is stable, otherwise this approach does not produce a control law of any practical use. This internal dynamics can be found by completing the coordinate transform, which leads to a partial differential equation (PDE).

In order to avoid coping with a PDE, which is rather complicated, we use a simplified method. To this end it is useful to know that the zero dynamics can be characterized in the original coordinates [6]. Noting that:

$$
y(t) \equiv 0 \Rightarrow \boldsymbol{y}(t)=\left[\begin{array}{lll}
y_{1}(t) & y_{2}(t) & y_{3}(t)
\end{array}\right]^{T} \equiv 0 \Rightarrow u(t) \equiv \frac{-f(\boldsymbol{x}(t))}{g(\boldsymbol{x}(t))}
$$

we see that if the output is identical to zero, the solution of the system equations must be confined to the smooth surface (manifold):

$$
Z=\left\{\boldsymbol{x}: x_{1}=0, \quad x_{2}=0, \quad x_{3}+c x_{4}=0\right\}
$$

and the input must be:

$$
u=u^{*}(\boldsymbol{x})=\frac{-f(\boldsymbol{x}(t))}{g(\boldsymbol{x}(t))}{ }_{\mid x \in Z}=\frac{c-b}{c} x_{4}
$$

Now, inserting the state coordinates defined by (14) and the control $u$ of (15) to the original system (4), we get the equation describing the zero dynamics in the form

$$
\dot{x}_{4}=-\frac{b}{c} x_{4}
$$

As the parameters $b$ and $c$ are positive, we have proved that the zero dynamics is asymptotically stable.

\section{NONLINEAR OBSERVER DESIGN}

For a practical application of formula (9), we have to measure the state vector $\mathrm{x}=\left[\begin{array}{llll}\mathrm{x}_{1} & \mathrm{x}_{2} & \mathrm{x}_{3} & \mathrm{x}_{4}\end{array}\right] \mathrm{T}=\left[\begin{array}{lll}\psi & \mathrm{r} \mathrm{x}_{3} & \delta\end{array}\right] \mathrm{T}$. As the access to the variable $\mathrm{x}_{3}$ can be problematic, a state observer must be used in order to overcome this difficulty.

We propose the following reduced-order, nonlinear observer:

$$
\left[\begin{array}{c}
\dot{\hat{x}}_{2} \\
\dot{\hat{x}}_{3}
\end{array}\right]=\left[\begin{array}{cc}
0 & 1 \\
0 & -a
\end{array}\right]\left[\begin{array}{l}
\hat{x}_{2} \\
\hat{x}_{3}
\end{array}\right]+\left[\begin{array}{c}
0 \\
-b
\end{array}\right] H\left(x_{2}\right)+\left[\begin{array}{l}
c \\
e
\end{array}\right] x_{4}+\left[\begin{array}{l}
l_{1} \\
l_{2}
\end{array}\right](y-\hat{y})
$$

$$
\hat{y}=\left[\begin{array}{ll}
1 & 0
\end{array}\right]\left[\begin{array}{l}
\hat{x}_{2} \\
\hat{x}_{3}
\end{array}\right]=\hat{x}_{2}
$$

where $y=x_{2}=r$ is assumed the measurable signal and the gain matrix $\mathrm{L}=[11,12] \mathrm{T}$ should be selected as to get the observation error (compare (23)) exponentially converging to the origin.

By means of (17) we can get the estimate $\hat{x}_{3}$.

The question is if putting into the controller (10) the estimate $\hat{\mathrm{X}}_{3}$ (from the observer (17)), instead of the original value $\mathrm{x}_{3}$, does not affect the system stability.

To prove that the overall system will be still stable, we perform the following reasoning.

Let us first re-write the formula (11) in the form:

$\dddot{y}=k_{1}\left(y-y_{d}\right)+k_{2} \dot{y}+k_{3} \ddot{y}=k_{1}\left(x_{1}-y_{d}\right)+k_{2} x_{2}+k_{3}\left(x_{3}+c x_{4}\right)$

Knowing now that instead of $\mathrm{x}_{3}$ we have in fact $\hat{\mathrm{x}}_{3}$, we re-write (18) as follows:

$$
\dddot{y}=k_{1}\left(x_{1}-y_{d}\right)+k_{2} x_{2}+k_{3}\left(\hat{x}_{3}+c x_{4}\right)
$$

Now adding to and subtracting from the left side of (19) the term $\mathrm{k}_{3} \mathrm{x}_{3}$ we get:

$$
\dddot{y}=k_{1}\left(x_{1}-y_{d}\right)+k_{2} x_{2}+k_{3}\left(x_{3}+c x_{4}\right)-k_{3}\left(x_{3}-\hat{x}_{3}\right)
$$

Denoting the estimation error as $\widetilde{\mathrm{x}}_{3}=\left(\mathrm{x}_{3}-\hat{\mathrm{x}}_{3}\right)$ we finally get:

$$
\dddot{y}-k_{3} \ddot{y}-k_{2} \dot{y}-k_{1}\left(y-y_{d}\right)=k_{3} \tilde{x}_{3}
$$

or

$$
\dddot{\tilde{y}}-k_{3} \ddot{\tilde{y}}-k_{2} \dot{\tilde{y}}-k_{1} \tilde{y}=k_{3} \tilde{x}_{3}
$$

where $\widetilde{y}=y-y_{d}$.

By subtracting from second and third equations of the system (4) the observer equations (17) we get the system describing observation error:

$$
\left[\begin{array}{c}
\dot{\tilde{x}}_{2} \\
\dot{\tilde{x}}_{3}
\end{array}\right]=\left[\begin{array}{cc}
l_{1} & 1 \\
l_{2} & -a
\end{array}\right]\left[\begin{array}{l}
\tilde{x}_{2} \\
\tilde{x}_{3}
\end{array}\right]
$$

Treating now $\widetilde{\mathrm{x}}_{3}$ as an output of the system (23) and knowing that it at the same time is the input to the system (22), we have a cascaded inter-connection of two asymptotically stable, linear time-invariant systems, which makes the overall system (Fig. 2) also asymptotically stable. 


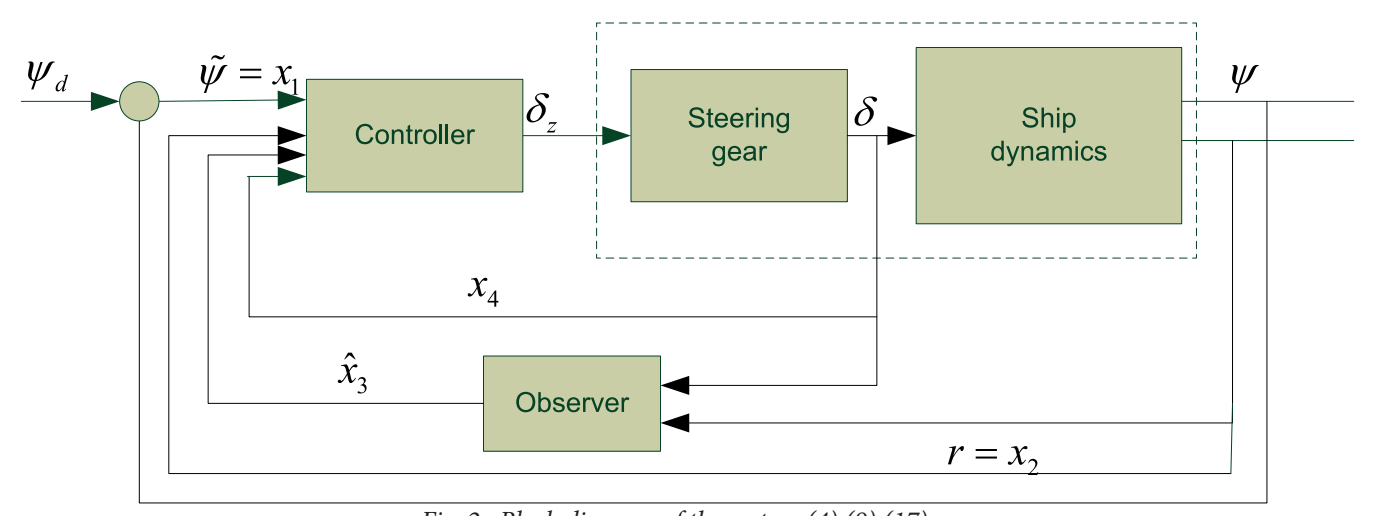

Fig. 2. Block diagram of the system (4),(9),(17)

\section{COURSE-CHANGING PROCESS SIMULATIONS}

The standard method of assessing the control system quality is based on analysis of the transition process as a response to the step input. Thus, in the following simulations we will test the ship behaviour after step-change of the course set-point for directionally stable or unstable ship.

The following charts show the situations where the ship is moving ahead at a steady speed $(0.25 \mathrm{~nm} / \mathrm{min})$ along a straight line, and then we apply a 30 -degree step course change command.

The graphs depicted in Fig.3 pertain to a directionally stable ship (coefficient $b_{1}=2$ ) and show respectively the plots of heading, rudder deflection versus time as well as the estimate $\hat{\mathrm{x}}_{3}$ derived from the state observer.

Corresponding plots are given in Fig. 4., however, they relate to the ship unstable on the course $\left(b_{1}=-2\right)$.

In the tests depicted in Fig. 3 it have been admitted some parametrical uncertainties. For example, the estimate of parameter $\mathrm{a}$, used in the controller, was taken as $\hat{a}=2 \mathrm{a}$ or the observer initial values were chosen as non-zero, i.e. $\hat{\mathrm{x}}_{2}(0)=0,5$; $\hat{\mathrm{x}}_{3}(0)=-0,5$.

In the case of Fig. 4., also certain robustness in respect to the parameter a may be observed. However, variations of the other parameters or/and observer initial values lead to the easy destabilization of the system. That is why in Fig. $4 \mathrm{c}$ the observer initial values were chosen equal to zero, which justify the plots overlapping. One interesting observation here is that, for the starboard ship turn, the rudder, after slight action to the right, is hardly deflected in the opposite direction (Fig.4b).

The remaining data, as the controller gains, are: $\mathrm{k}_{1}=-6$; $\mathrm{k}_{2}=-11 ; \mathrm{k}_{3}=6$ - for the stable ship or $\mathrm{k}_{1}=-3 ; \mathrm{k}_{2}=-6,5 ; \mathrm{k}_{3}=-4,5$ - for the alternative case. The observer gains are, in both cases, chosen as $-l_{1}=5,9 ; 1_{2}=8,3$.

Although the simulation tests look quite well, we should be aware that it is the result of the (nearly) full knowledge of the model parameters used by the controller.

While the first case indicates a certain degree of robustness, in the second case (the directionally unstable ship) there is significant susceptibility to all possible uncertainties, such as: parametrical uncertainties, the choice of observer initial conditions, steering machine saturation, etc. The relatively small variations in each of them may lead to system destabilization (a)

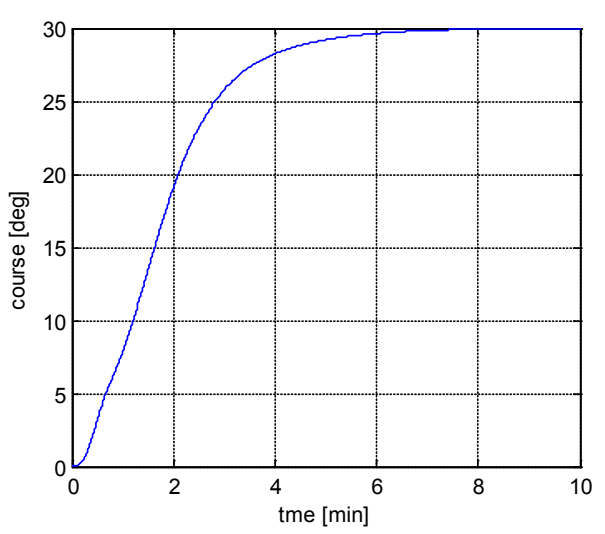

(b)

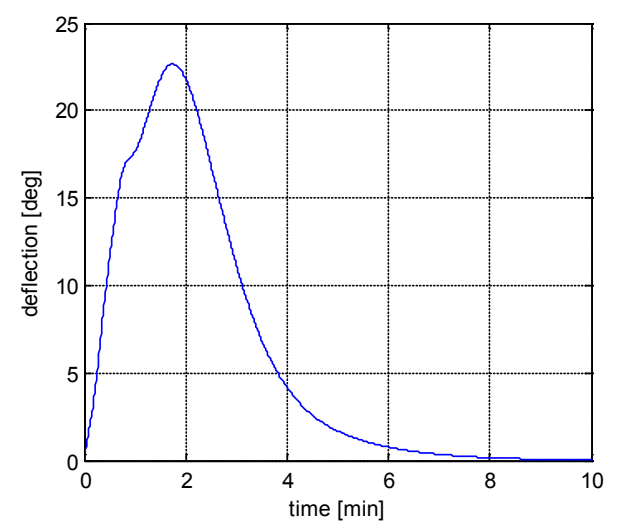

(c)

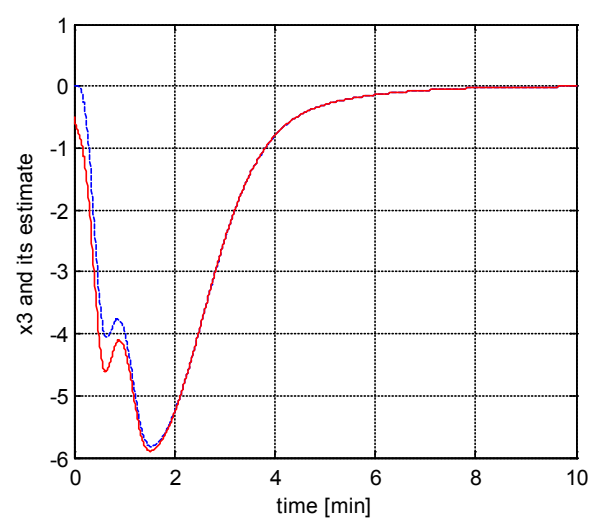

Fig.3. The plots of: a) ship headings, b) rudder deflection, c) state coordinate estimation (red solid line) versus its exact value - for the directionally stable ship 
(a)

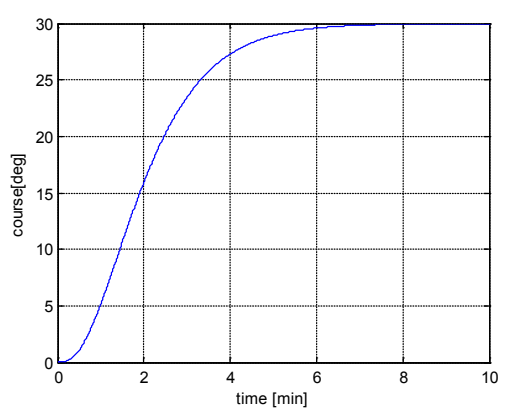

(b)

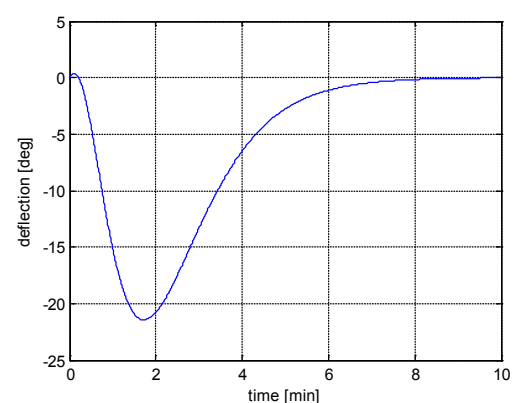

(c)

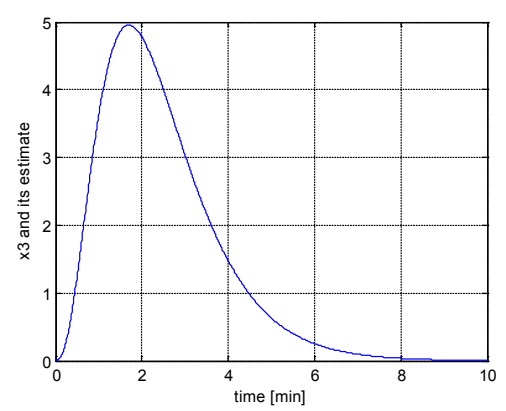

Fig.4. The plots of: a) ship headings, b) rudder deflection, c) state coordinate estimation (red solid line) versus its exact value - for the directionally unstable ship

destroying the practical usability of the proposed controller. That is why this case should be treated with special care.

In order to make the controller practically applicable, the adaptive or robust versions of its design are extremely important. In this respect this work is only a theoretical introduction to further research in the field.

\section{CONCLUSIONS}

The author deals with the problem of ship autopilot design based on a relatively complex, nonlinear dynamical model of the vessel. This leads to the need of using advanced theoretical methods of nonlinear control including the theory of non-linear observers. It has been proven that the obtained resultant system as the cascaded inter-connection of the original system (the ship and controller) and the observer is asymptotically stable.

This article is an introduction that provides some theoretical basis for further engineering design work of ship control systems, based on the complex, Bech's non-linear ship model.

In view of the fact that for its practical applicability we also have to take into account the system parametrical uncertainties, this article is the first part of a larger project.

\section{BIBLIOGRAPHY}

1. van Amerongen J.: Adaptive steering of ships. A modelreference approach to improved manoeuvring and economical course keeping. Ph.D. Thesis, Delft University of Technology, 1982.

2. Control System Toolbox User's Guide. For Use with MATLAB. The MathWorks, Inc. 1998.

3. Chislett, M. and Strom-Tejsen, I. : Planar Motion Mechanism Tests and Full-Scale Steering and Maneuvering Predictions for Mariner Class Vessel, Technical Report Hy-5, Hyro and Aerodynamics Laboratory, Lyngby, Denmark, 1965.

4. Fossen T. I.: Guidance and control of ocean vehicles. John Wiley, Chichester, USA, 1994.

5. Isidori A.: Nonlinear Control Systems. An introduction, Springer -Verlag, Berlin, 1989.

6. Khalil H.K.: Nonlinear Systems. Prentice Hall, Upper Saddle River, NJ, 2002.

7. Krstic, M., Kanellakopoulos, I. and Kokotovic, P. Nonlinear and Adaptive Control Design, Wiley-Interscience, New York, 1995.

8. Lewis F. W., Jagannathan S. and A. Yesildirak: Neural Network Control of Robot Manipulators and Non-Linear Systems. Taylor \& Francis, 1998.

9. Lisowski J.: Ship as an object of automatic control ( in Polish), Wyd. Morskie, Gdańsk 1981

10. Márquez H. J.: Nonlinear control systems. Analysis and design. John Wiley, NY, 2003

11. Morawski L., Pomirski J.: Design of the robust PID course-keeping control system for ships. Polish Maritime Research, No. 1, 2002.

12. Sastry, S. and Isidori, A. Adaptive Control of Linearizable Systems. IEEE Transactions on Automatic Control 34(11),1989, pp. 1123-1131

13. Slotine, JE. and Weiping Li. Applied Nonlinear Control. Prentice Hall, Englewood Cliffs, NY, 1991.

14. Zwierzewicz Z.: Ship course-keeping by nonlinear adaptive control synthesis. Int. Journal of Factory Automation, Robotics and Soft Computing, 2007, No. 2, April, 2007, pp.102-107. 
15. Zwierzewicz Z.: Methods and algorithms of ship automatic control systems (in Polish). Scientific publishing house of Szczecin Maritime University, Szczecin 2012.

\title{
CONTACT WITHE THE AUTOR
}

\section{Zenon Zwierzewicz}

\author{
Maritime University of Szczecin \\ 1-2 Wały Chrobrego St. \\ 70-500 Szczecin, Poland
}

